\title{
Prevalence of the metabolic syndrome in patients with hypertension treated in general practice in Spain: an assessment of blood pressure and low-density lipoprotein cholesterol control and accuracy of diagnosis
}

\author{
Vivencio Barrios, Carlos Escobar, Alberto Calderón, José L. Llisterri, Eduardo Alegría, \\ Javier Muñiz, Arantxa Matalí
}

\begin{abstract}
This study was designed to evaluate whether primary care physicians in Spain accurately diagnose the metabolic syndrome in hypertensive patients, to define the profile and management of these patients in clinical practice, and to ascertain the level of blood pressure and low-density lipoprotein cholesterol control. Data were analyzed from a cross-sectional survey involving 12,954 patients with hypertension (Prevención Cardiovascular en España en Atención Primaria: Intervención Sobre el Colesterol en Hipertensión [PRESCOT] study), wherein 52\% of the cohort fulfilled the National Cholesterol Education Program-Adult Treatment Panel criteria for the metabolic syndrome. The majority of patients $(54.6 \%$ ) had 3 risk factors, $32.4 \%$ had 4 , and $13 \%$ had 5 risk factors. Physician diagnosis of the metabolic syndrome was poor, with $43.7 \%$ of physicians missing the diagnosis and $12.9 \%$ wrongly diagnosing the metabolic syndrome. Blood pressure and low-density lipoprotein cholesterol control rates were very low, with only 4.7\% of metabolic syndrome patients achieving control for both blood pressure and low-density lipoprotein cholesterol vs $13.5 \%$ for non-metabolic syndrome patients $(\mathrm{P}<.0001)$. These findings demonstrate that the metabolic syndrome is common in patients with hypertension and that it is generally poorly diagnosed and treated by primary care physicians.
\end{abstract}

The metabolic syndrome (MetS) is characterized by an array of cardiovascular risk factors that may be predictive of longer-term disease sequelae in certain individuals. The syndrome is associated with insulin resistance and hyperglycemia and coincides with an increased risk of developing type 2 diabetes and cardiovascular disease. ${ }^{1-5}$ Although Reaven ${ }^{6}$ described the syndrome in the late 1980s, a precise definition and the clinical importance of MetS are still evolving. In the United States, the Third Report of the National Cholesterol Education Program (NCEP) Expert Panel on Detection, Evaluation, and Treatment of High Blood Cholesterol in Adults (ATP III) ${ }^{7}$ has provided a relatively simple definition of MetS. NCEP-ATP III criteria are widely used in clinical practice, and they facilitate an easy identification of the syndrome with uncomplicated criteria that can be applied in the most basic health care setting. Recently, the International Diabetes Federation (IDF) proposed a definition that emphasizes the importance of abdominal obesity and lowers the threshold for several parameters such as waist circumference, blood pressure (BP), and fasting plasma glucose. ${ }^{8}$

As diabetes and obesity in Western countries are increasing, almost to epidemic proportions, not surprisingly the prevalence of MetS is progressively growing. ${ }^{9}$ Without intervention this will almost certainly result in an increase in cardiovascular morbidity and place a greater burden on Western society. $^{10,11}$

Although MetS is associated with cardiovascular disease, the risk is not homogeneous and may vary according to age, sex, and severity. ${ }^{12,13}$ Patients with hypertension and MetS represent a particularly highrisk group, not only because of the higher prevalence of target organ damage, ${ }^{14}$ but also because of the increased incidence of cardiovascular complications observed in this population. ${ }^{12,15}$ The presence of MetS in hypertensive patients acquires greater clinical relevance and, to improve prognosis, it is important to be able to accurately diagnose MetS, to better understand the long-term impact, and to reduce the number and severity of risk factors. BP and low-density lipoprotein cholesterol (LDL-C) are established risk factors that are strongly associated with cardiovascular morbidity and mortality, and longer-term control of these will likely significantly improve the survival of MetS patients. ${ }^{16}$ 
MetS has been associated with an increased risk for cardiovascular disease in a number of pivotal trials, including community-based populations, and in primary preventive settings. ${ }^{1}$ Studies involving large numbers of patients with hypertension and MetS treated in Spanish general practice facilities are not so common. Furthermore, few data are available regarding the ability of primary care physicians to accurately diagnose MetS. The goals of this general practice study undertaken in Spain were to:

- $\quad$ Determine the prevalence of MetS according to the NCEP-ATP III definition;

- Determine accuracy in physician diagnosis of MetS;

- -Determine the demographics of and medications used in patients with MetS compared with those without MetS; and

- $\quad$ Compare control rates of BP and lipids in patients with and without MetS.

\section{Patients and Methods}

Study Population.

The study population involved patients with hypertension included in the Prevención Cardiovascular en España en Atención Primaria: Intervención Sobre el Colesterol en Hipertensión (PRESCOT) crosssectional epidemiologic survey. The aim was to determine the clinical profile and associated risk factors, including MetS, in a population of patients with hypertension attending general practice clinics across Spain. Approximately 2000 primary care physicians participated in the study, which was performed during the first quarter of 2004. Each investigator was asked to include the first 6 patients with hypertension who attended their outpatient clinic. Inclusion criteria were age 18 years and older, man or woman, hypertension (systolic BP $>140 \mathrm{~mm} \mathrm{Hg}$ and/or diastolic BP $>90 \mathrm{~mm} \mathrm{Hg}$ or receiving antihypertensive treatment), and an available blood test with a complete lipid profile within the past 6 months. To best reflect clinical practice in Spain, no specific exclusion criteria were defined.

\section{Methodology and Data Collection.}

Seated BP was measured after 5 minutes of resting. Two measurements were taken and the mean was recorded. BP was considered to be well controlled using the guidelines of the European Society of Hypertension ( $<140 / 90 \mathrm{~mm} \mathrm{Hg}$ for the general population and $<130 / 80 \mathrm{~mm} \mathrm{Hg}$ for diabetic patients). ${ }^{15}$

Patients were classified as dyslipidemic when they had a confirmed diagnosis of an impaired lipid profile (hypercholesterolemia, hypertriglyceridemia, or mixed hyperlipidemia) according to NCEP-ATP III or were receiving lipid-lowering therapy.

Coronary risk stratification was performed using the NCEP-ATP III classification. ${ }^{7}$ High-risk patients were those with coronary disease or a risk equivalent (10-year coronary risk $>20 \%)$, medium-risk patients were those with 2 or more risk factors (10-year coronary risk 10\%-20\%), and low-risk patients were those with 1 or no cardiovascular risk factors (10-year coronary risk $<10 \%)$. Coronary risk equivalents included peripheral arterial disease, abdominal aortic aneurysm, carotid disease, and diabetes. Risk factors included smoking, high BP, decreased levels of high-density lipoprotein cholesterol (HDL-C) ( $<40 \mathrm{mg} / \mathrm{dL}$ ), family history of premature coronary disease (first-degree relatives younger than 55 years in men and younger than 65 years in women), and age ( 45 or older in men and 55 and older in women). ${ }^{7}$ LDL-C control was thus defined in relation to the above risk categories as established by NCEP-ATP III guidelines: LDL-C $<100 \mathrm{mg} / \mathrm{dL}$ for the high-risk group, $<130 \mathrm{mg} / \mathrm{dL}$ for the medium-risk group, and $<160 \mathrm{mg} / \mathrm{dL}$ for the low-risk group.

MetS was diagnosed using NCEP-ATP III criteria requiring the presence of 3 or more of the following: abdominal obesity (waist circumference $>102 / 88 \mathrm{~cm}$ or $>40 / 35$ inches for men/women); triglycerides $\geq 150 \mathrm{mg} / \mathrm{dL}$; HDL-C $<40 / 50 \mathrm{mg} / \mathrm{dL}$ (men/women); fasting glucose $\geq 110 \mathrm{mg} / \mathrm{dL}$; or BP $\geq 130 / 85 \mathrm{~mm} \mathrm{Hg}{ }^{7}$ Waist circumference was measured at the midway point between the iliac crest and the costal margin. NCEP-ATP III criteria were used to define MetS because of their ease in applying in primary care, which has caused this definition to be the most utilized by general practitioners in Spain. The physicians completed a specific individual case report form with all the collected clinical and analytic data. They were asked to identify the presence of MetS according ATP III criteria, and the diagnosis was specifically recorded in the case report form. To facilitate the physicians' task, a sheet with the ATP III diagnostic criteria was included in the study documentation. A post hoc analysis was performed using the IDF criteria. ${ }^{8}$

To evaluate whether primary care physicians correctly identified MetS, their diagnosis was compared with an independent control assessment using patient data stored on a central database. 


\section{Statistical Analyses.}

Various statistical tests were performed depending on the nature of variables being compared. The chi-square test was used to analyze the relationship between categoric variables; however, when $>20 \%$ of the cells had an expected frequency lower than 5, the Fisher exact test was employed. Comparison of continuous variables between groups was performed using the Student $t$ test. Database design was subjected to internal consistency rules and ranges to control inconsistencies/inaccuracies in the collection and tabulation of data (SPSS version 11.0.1; SPSS Inc, Cary, NC).

\section{Results}

The PRESCOT survey enrolled a total of 15,707 patients, of which 12,954 (82.5\%) were finally included in the analysis after excluding those who did not comply with the selection criteria and/or whose case report forms were incomplete and/or inconsistent. Of the 12,954 patients included in the survey, $5866(52 \%)$ fulfilled NCEP-ATP III diagnostic criteria for MetS. Of these, $54.6 \%$ had the minimum of 3 MetS risk factors, $32.4 \%$ had 4 risk factors, and $13 \%$ had 5 risk factors. Interestingly, if the newer IDF classification $^{8}$ was applied, the prevalence of MetS in our hypertensive population would have risen from $52 \%$ to $75.5 \%$.

Table I details the baseline characteristics of the overall study population subdivided into patients with MetS and patients without MetS. All risk factors for cardiovascular disease relating to BP control and hyperlipidemia were significantly worse in the MetS group (higher systolic and diastolic BP, total cholesterol, LDL-C and triglycerides, and lower HDL-C levels). Interestingly, there were more women than men in the MetS group (56.1\% vs $43.9 \%$, respectively; $P<.0001)$. Excluding hypertension, the most common components of MetS were abdominal obesity (as measured by waist circumference; $50.5 \%$ of cases) and increased triglyceride levels (49.8\% of cases). There were some significant sex-related distribution differences in the patients with MetS (Table II), with more women than men presenting with abdominal obesity (59.6\%vs $41.4 \%$, respectively; $P<.0001)$ and low HDL-C levels $(40.3 \%$ vs $18.7 \%$, respectively; $P<.0001)$ and more men than women presenting with hypertriglyceridemia $(56.7 \%$ vs $43.4 \%$, respectively; $P<.0001)$.

Table I. Characteristics of Patients With Hypertension in the PRESCOT Trial by Presence or Absence of the Metabolic Syndrome (MetS)

\begin{tabular}{|c|c|c|c|}
\hline \multirow[b]{2}{*}{ Variables } & \multicolumn{2}{|c|}{ Demographic Data } & \multirow[b]{2}{*}{$P$} \\
\hline & Hypertensive Patients With MetS & Hypertensive Patients Without MetS & \\
\hline Sex, male/female, $\%$ & $43.9 / 56.1$ & $55.5 / 44.5$ & $<.0001^{*}$ \\
\hline Age, y & $62.3 \pm 10.5$ & $62.1 \pm 10.8$ & $.5161 \dagger$ \\
\hline BMI, $\mathrm{kg} / \mathrm{m}^{2}$ & $29.6 \pm 4.3$ & $27.3 \pm 3.7$ & $<.0001 \dagger$ \\
\hline Waist circumference, $\mathrm{cm}$ & $104.9 \pm 15.4$ & $93.4 \pm 14.3$ & $<.0001 \dagger$ \\
\hline $\mathrm{SBP}, \mathrm{mm} \mathrm{Hg}$ & $145.8 \pm 14.3$ & $142.2 \pm 13.8$ & $<.0001 \dagger$ \\
\hline DBP, mm Hg & $86.0 \pm 9.1$ & $83.9 \pm 8.9$ & $<.0001 \dagger$ \\
\hline Total cholesterol, mg/dL & $237.3 \pm 40.9$ & $227.3 \pm 42.2$ & $<.0001 \dagger$ \\
\hline Triglycerides, $\mathrm{mg} / \mathrm{dL}$ & $192.3 \pm 92.2$ & $120.1 \pm 46.6$ & $<.0001 \dagger$ \\
\hline LDL cholesterol, mg/dL & $154.2 \pm 93.5$ & $144.8 \pm 76.0$ & $<.0001 \dagger$ \\
\hline HDL cholesterol, mg/dL & $47.8 \pm 17.0$ & $58.5 \pm 16.4$ & $<.0001 \dagger$ \\
\hline Fasting plasma glucose, $\mathrm{mg} / \mathrm{dL}$ & $124.4 \pm 37.7$ & $96.8 \pm 16.7$ & $<.0001 \dagger$ \\
\hline
\end{tabular}

PRESCOT indicates the Prevención Cardiovascular en España en Atención Primaria Intervención Sobre el Colesterol en Hipertensión study; BMI, body mass index; SBP, systolic blood pressure; DBP, diastolic blood pressure; LDL, low-density lipoprotein; and HDL, high-density lipoprotein. ${ }^{*}$ Chi-square test. $†$ Student $t$ test. 
Table II. Prevalence of the Different ATP III Components in Patients With the Metabolic Syndrome

\begin{tabular}{|c|c|c|c|c|}
\hline Risk Factor & Total, \% & Men, $\%$ & Women, \% & $p^{*}$ \\
\hline Hypertension $(\mathrm{BP} \geq 130 / 85 \mathrm{~mm} \mathrm{Hg}$ or $\mathrm{Rx})$ & 100 & 100 & 100 & - \\
\hline Waist circumference $>102 \mathrm{~cm}$ for men or $>88 \mathrm{~cm}$ for women & 50.5 & 41.4 & 59.6 & $<.0001$ \\
\hline Triglycerides $\geq 150 \mathrm{mg} / \mathrm{dL}$ & 49.8 & 56.7 & 43.4 & $<.0001$ \\
\hline Low HDL cholesterol $<40 \mathrm{mg} / \mathrm{dL}$ for men or $<50 \mathrm{mg} / \mathrm{dL}$ for women & 29.5 & 18.7 & 40.3 & $<.0001$ \\
\hline Fasting plasma glucose $>110 \mathrm{mg} / \mathrm{dL}$ & 35.6 & 35.7 & 35.5 & .8079 \\
\hline
\end{tabular}

ATP III indicates the third report of the Adult Treatment Panel; BP, blood pressure; Rx, receiving antihypertensive treatment; and HDL, high-density lipoprotein. *Student $t$ test for continuous variables; chi-square test for categoric variables.

The $98.2 \%$ of patients with MetS were taking at least 1 antihypertensive drug; $17.7 \%$ were on monotherapy and $80.5 \%$ were treated with 2 or more drugs. MetS patients were taking significantly more antihypertensive medication than those without MetS $(45.3 \%$ vs $36.6 \%$ were on combined therapy, respectively; $P<.001)$. The use of angiotensin-converting enzyme inhibitors and angiotensin receptor blockers (74.8\% vs $67.6 \%$, respectively; $P<.001)$ and statins $(43.0 \%$ vs $39.1 \%$, respectively; $P<.001)$ was more frequent in MetS patients. Table III represents the percentage of antihypertensive and lipid-lowering drugs used in patients according to the presence of MetS.

Table III. Percentage of Antihypertensive and Lipid-Lowering Drugs Used in Patients According to the Presence of the Metabolic Syndrome (MetS)

\begin{tabular}{lcc}
\hline Type Of Treatment & Hypertensive Patients With MetS, $\%$ & Hypertensive Patients Without MetS, \% \\
\hline & & \\
Antihypertensive drugs & 43.5 & 39.6 \\
ACEIs & 38.3 & 32.8 \\
Diuretics & 32.7 & 28.7 \\
ARBs & 20.3 & 17.7 \\
Calcium antagonists & 12.7 & 13.0 \\
$\beta$-Blockers & 74.8 & 67.6 \\
ACEI and ARB & 4 & 3.5 \\
Other & & \\
Lipid-lowering drugs & 43.0 & 39.1 \\
Statins & 5.8 & 2.6 \\
Other & & \\
\end{tabular}

ACEI indicates angiotensin-converting enzyme inhibitor; ARB, angiotensin receptor blocker.

The diagnosis concordance between physicians and a control assessment was $56.3 \%$ for the presence of MetS and $87.1 \%$ for the absence of MetS (Figure 1). Crucially, general practitioners did not diagnose $43.7 \%$ of cases of MetS and $12.9 \%$ of patients were wrongly diagnosed as having MetS despite not meeting NCEP-ATP III criteria. 


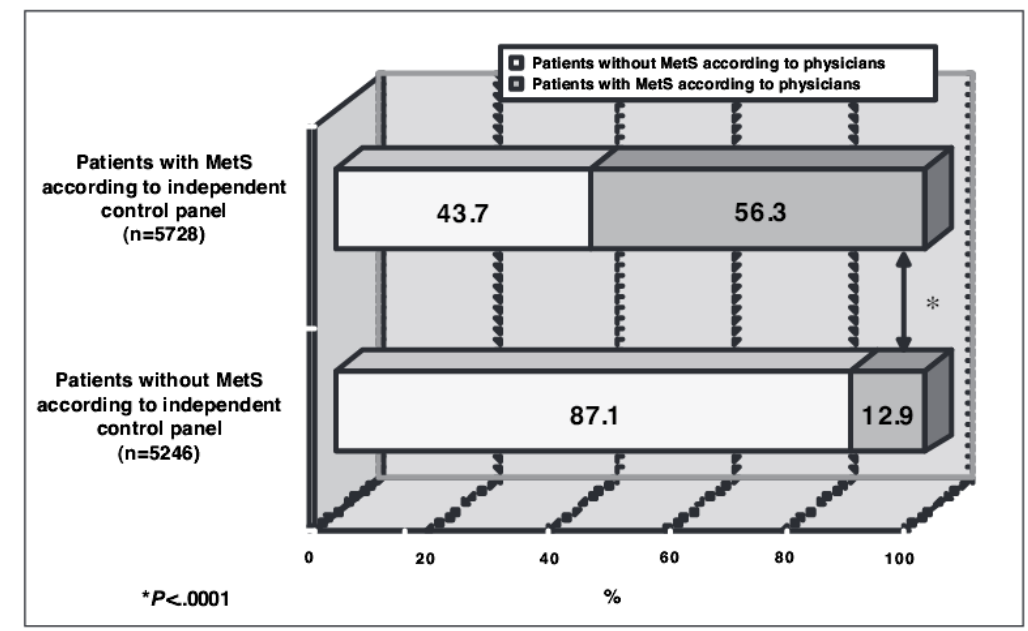

Figure 1. Diagnosis of the metabolic syndrome (MetS) by primary care physicians compared with independent assessment.

Findings with regard to BP and LDL-C control are presented in Figure 2. BP was controlled in fewer patients with MetS compared with those without MetS (17.2\% vs 33.6\%, respectively; $\mathrm{P}<.001)$. Systolic BP was controlled in $20.7 \%$ of MetS patients and in $37.2 \%$ of non-MetS patients $(\mathrm{P}<.0001)$, while diastolic BP was controlled in $39.0 \%$ vs $63.7 \%$ ( $\mathrm{P}<.0001$ ), respectively. Similarly, LDL-C control was worse in MetS patients than in patients without MetS (17.2\% vs $35.7 \%$, respectively; $\mathrm{P}<.0001)$. As expected, the degree of control of BP and LDL-C combined was also lower in patients with MetS $(4.7 \%$ vs $13.5 \% ; \mathrm{P}<.0001)$.

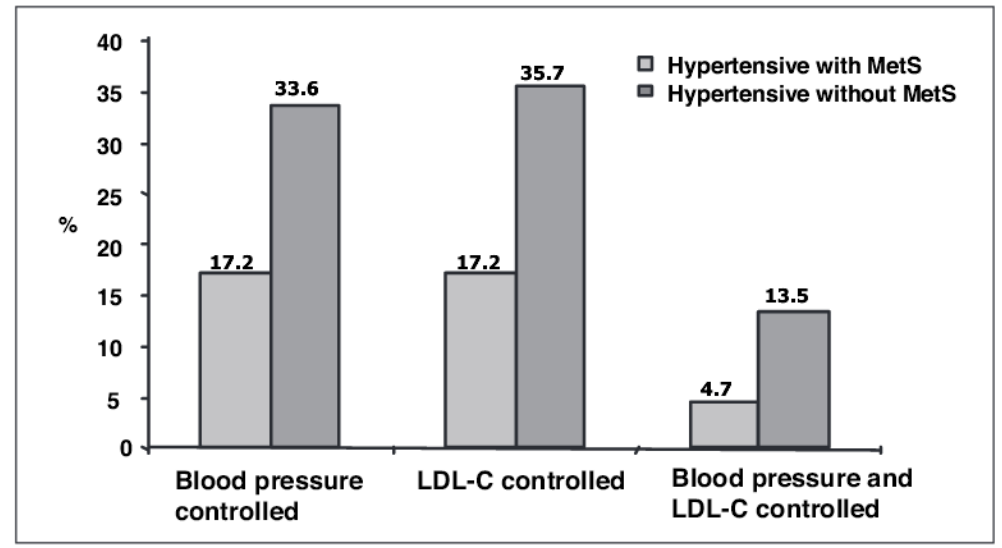

Figure 2. Degree of blood pressure and low-density lipoprotein cholesterol (LDL-C) control in hypertensive patients according to the presence of the metabolic syndrome (MetS).

Among the patients with MetS, the BP and LDL-C controls were worse in the subgroup of MetS patients with diabetes compared with nondiabetics (Table IV). BP was controlled in $23.0 \%$ of MetS patients without diabetes and in $9.5 \%$ of MetS patients with diabetes $(P<.001)$. Similar results were observed for LDL-C, control being achieved in $22.2 \%$ of MetS nondiabetic patients and $10.5 \%$ of MetS patients with diabetes $(P<.0001)$. The combined BP and LDL-C control was achieved in $6.3 \%$ of nondiabetic patients with MetS and in only $2.6 \%$ of diabetics with MetS $(P<.001)$. 
Table IV. Percentage of Patients With Hypertension and Coexisting Metabolic Syndrome (MetS) (With or Without Diabetes) Achieving Control of BP and LDL-C

\begin{tabular}{lccr}
\hline Variables & $\begin{array}{c}\text { Hypertensive Patients With MetS and } \\
\text { Diabetes, \% }\end{array}$ & $\begin{array}{c}\text { Hypertensive Patients With MetS Without } \\
\text { Diabetes, \% }\end{array}$ & \multicolumn{2}{c}{$p^{*}$} \\
\hline & 9.5 & 23.0 & $<.0001$ \\
$\begin{array}{l}\text { BP controlled } \\
\text { LDL-C controlled }\end{array}$ & 10.5 & 22.2 & $<.0001$ \\
$\begin{array}{l}\text { Both BP and LDL-C } \\
\text { controlled }\end{array}$ & 2.6 & 6.3 & $<.0001$ \\
\hline
\end{tabular}

BP indicates blood pressure; LDL-C, low-density lipoprotein cholesterol.*Student $t$ test for continuous variables; chi-square test for categoric variables.

\section{Discussion}

A number of pivotal studies have been reported in the past 5 years that have investigated the prevalence of MetS. One of the pioneering studies in this regard was the third report of the National Health and Nutrition Examination Survey (NHANES III) study, ${ }^{17}$ which randomly selected 8800 Americans between the ages of 20 and 89 years. The overall prevalence of MetS in this survey was $21.8 \%$, and studies such as Framingham, ${ }^{18}$ West of Scotland Coronary Prevention Study (WOSCOPS), ${ }^{19}$ the Women's Health Study, ${ }^{20}$ and other studies carried out in Western countries ${ }^{3-5}$ have reported similar prevalence rates.

Few studies have been carried out in Spain investigating MetS. Martínez-Larrad and colleagues ${ }^{21}$ reported a prevalence of $17 \%$ in urban and rural areas using NCEP-ATP III criteria. A prevalence of 24.4\% was observed in the Canary Islands, and the rate increased with age and decreased with educational level. ${ }^{22}$ In a primary care study, Ascaso and colleagues ${ }^{23}$ found that MetS prevalence was $48.4 \%$ in subjects with abdominal obesity compared with $18.8 \%$ in those without abdominal obesity. A large study that included more than 19,000 workers recorded a MetS prevalence of $12 \%$ (16\% in men and $8 \%$ in women). ${ }^{24}$ Clear geographic differences regarding the distribution of MetS have been detected, and these are probably a consequence of different environmental factors such as diet and physical exercise. More recently, de la Sierra and associates ${ }^{25}$ reported a prevalence of $44.6 \%$ using NCEP-ATP III criteria and $61.7 \%$ using IDF criteria in a primary care study in Spain involving 19,039 patients with hypertension. The results of our large cross-sectional survey involving hypertensive patients attending primary health care centers in Spain demonstrated an even higher prevalence of MetS: $52 \%$ using NCEPATP III criteria and $75.5 \%$ using IDF criteria. In our series, the presence of MetS was more frequent in women $(56.1 \%$ vs $43.9 \%$ for men; $P<.0001)$. It should be taken into account that this study was carried out in Spanish primary care; therefore, these results could somehow differ in other sites and may not be generalizable to other countries with different health care delivery.

It is notable that despite being American, the NCEP-ATP III criteria have been widely accepted in Spain. It is probably because NCEP-ATP III criteria are easy to apply, which results in the MetS being easily diagnosed in primary care. Although the NCEP-ATP III guidelines define MetS as the presence of 3 or more diagnostic criteria, the risk is not homogeneous and may vary according to age, sex, and severity of MetS. ${ }^{12,13}$ In the current study, almost half of the MetS patients (45.5\%) had 4 or 5 diagnostic criteria, which is indicative of high cardiovascular risk. ${ }^{12}$ Furthermore, this study was performed in a hypertensive cohort, which, in itself, confers added risk. Therefore, it is not surprising that a higher prevalence of MetS was detected in this study compared with general population studies and is more in line with findings from trials involving patients with diabetes or heart disease. For example, a prevalence of $77 \%$ was noted in a study involving 318 type 2 diabetics $^{26}$ and a rate of $41.1 \%$ in patients with heart disease. $^{27}$

Japanese researchers reported a positive correlation between MetS prevalence and BP in a study involving 506 untreated hypertensive patients. ${ }^{28}$ Patients were divided into 3 groups according to the Seventh Report of the Joint National Committee on Prevention, Detection, Evaluation, and Treatment of High Blood Pressure (JNC 7) classification and the prevalence of MetS was $9.9 \%$ in normotensive subjects, $19.2 \%$ in prehypertensive patients, and $35.5 \%$ in the hypertensive subjects. A positive correlation between insulin resistance and BP was also documented, and the authors noted that the higher the $\mathrm{BP}$, the greater the number of risk factors for MetS. A somewhat higher prevalence of 37.6\% for MetS in prehypertensive patients was recently reported in the Trial for Preventing Hypertension (TROPHY) 
substudy. ${ }^{29}$ With regard to MetS prevalence, our study confirms the finding of Ford ${ }^{30}$ who reported that the rate is significantly higher when IDF criteria are used.

Evaluation of the quality of diagnosis of MetS in the current trial showed that physician diagnosis of MetS was generally poor: in $43.7 \%$ of cases they missed the diagnosis and in $12.9 \%$ they wrongly diagnosed MetS when diagnostic criteria were not met. These data highlight the need for continuous medical education to improve the management of this syndrome in general practice. Previous studies had noted an underestimation of the cardiovascular risk in this setting. ${ }^{31,32}$ Thus, in the Estratificación del Riesgo Cardiovascular en Hipertensión en Atención Primaria e Impacto Sobre el Tratamiento Antihipertensivo (DIORISC) study, ${ }^{31}$ which included 9374 hypertensive patients managed in primary care, $61 \%$ of patients belonged to a high or very high-risk group, yet physicians only identified $39 \%$ of these patients as being at high or very high risk $(P<.0001) .{ }^{31}$

There is now a body of evidence demonstrating that MetS increases cardiovascular morbidity and mortality, reinforcing the importance of optimizing management of these patients and achieving adequate control of risk factors. ${ }^{16}$ Furthermore, in hypertensive patients with MetS, cardiovascular morbidity and mortality are significantly higher and so the relative risk is compounded. Schillaci and colleagues ${ }^{12}$ observed that hypertensive patients with MetS had double the number of cardiovascular events compared with patients without MetS. They also noted that the greater the number of MetS risk factors, the worse the prognosis.

The results of the current study provide an insight into the status of the treatment of patients with hypertension and coexisting MetS in general practice and highlight some important concerns regarding the management of cardiovascular risk. Indeed, the overall control of hypertension and dyslipidemia was relatively poor in this cohort. The proportion of patients achieving control of both BP and LDL-C was low for the total patient population, but it was worse in patients with MetS than in those without MetS (4.7\% vs $13.5 \%$,respectively; $P<.0001)$ and worse still in hypertensive patients with MetS and diabetes (2.6\%). These results indicate that in patients with hypertension, management of BP and LDL-C and risk factors for cardiovascular disease is generally very poor in Spain. Management is worse in patients with coexisting MetS and, interestingly, this occurs despite the fact that patients with MetS were receiving more medications to reduce both BP and LDL-C than patients without MetS and were also receiving more antihypertensive drugs than was reported in previous studies..$^{31,33}$

The cross-sectional design of the study was chosen to best represent real-world clinical practice. Consequently, a large population of hypertensive patients was included in the trial with very few patient exclusion criteria. This was achieved by consecutive sampling. This methodology has its limitations since it reduces the level of control that can be exercised to reduce variation and bias (eg, random sampling and "blind" controls). The large number of patients included in the study, however, and the nature of the end points being measured, with no comparators under review, minimizes this theoretical limitation. It is notable that, since this study was performed in primary care in Spain, the results may not be generalizable to other countries with different health care delivery.

Overall, the study was designed to represent clinical practice, presenting an accurate picture of the hypertensive population attending primary care.

\section{Conclusions}

These data demonstrate the high prevalence of MetS in a hypertensive population attending primary care facilities in Spain. More than half of the patients fulfilled NCEP-ACT III criteria for MetS. Management of BP and LDL-C, key risk factors for cardiovascular morbidity and mortality, was worse in patients with MetS than in those without the syndrome.

Although interest in MetS has recently increased, it is not always accurately identified. The findings of this study show that it is clearly underdiagnosed and undertreated. This highlights the need for ongoing medical education to improve the management of this syndrome in general practice, with the goal of aggressively reducing cardiovascular risk.

\section{Acknowledgments and disclosures}

The authors wish to express their sincere gratitude to all investigators who actively participated in this study. Without their dedication and quality of work, the present publication would not have been possible. Unfortunately, the list of investigators is too long to include in this publication. We would also like to acknowledge Almirall-Prodesfarma Pharmaceuticals for its financial support and Biometrica for the statistical analyses, both indispensable to complete this observational study. 


\section{References}

1. Sundstrom J, Riserus U, Byberg L, et al. Clinical value of the metabolic syndrome for long term prediction of total and cardiovascular mortality: prospective, population based cohort study. BMJ. 2006;332:878-882.

2 Haffner SM. Risk constellations in patients with the metabolic syndrome: epidemiology, diagnosis, and treatment patterns. Am J Med. 2006;119:S3-S9.

3 Lakka HM, Laaksonen DE, Lakka TA, et al. The metabolic syndrome and total and cardiovascular disease mortality in middle-aged men. JAMA. 2002;288:2709-2716.

4 Alexander CM, Landsman PB, Teutsch SM, et al. NCEP defined metabolic syndrome, diabetes, and prevalence of coronary heart disease among NHANES III participants age 50 years and older. Diabetes. 2003;52:1210-1214.

5 Hunt KJ, Resendez RG, Williams K, et al. National Cholesterol Education Program versus World Health Organization metabolic syndrome in relation to all-cause and cardiovascular mortality in the San Antonio Heart Study. Circulation. 2004;110:1251-1257.

6 Reaven GM. Role of insulin resistance in human disease. Banting lecture 1988. Diabetes. 1988;37:1595-1607.

7 National Cholesterol Education Program (NCEP) Expert Panel on Detection, Evaluation, and Treatment of High Blood Cholesterol in Adults (Adult Treatment Panel III). Third Report of the National Cholesterol Education Program (NCEP) Expert Panel on Detection, Evaluation, and Treatment of High Blood Cholesterol in Adults (Adult Treatment Panel III): final report. Circulation. 2002;106:3143-3421.

8 Alberti KG, Zimmet PZ, Shaw JE, for the IDF Epidemiology Task Force Consensus Group. The metabolic syndrome - a new world-wide definition from the International Diabetes Federation consensus. Lancet. 2005;366:1059-1062.

9 Zimmet P, Alberti KG, Shaw J. Global and societal implications of the diabetes epidemic. Nature. 2001;414:782787.

10 Eckel RH, Grundy SM, Zimmet PZ. The metabolic syndrome. Lancet. 2005;365:1415-1428.

11 Cameron AJ, Shaw JE, Zimmet PZ. The metabolic syndrome: prevalence in worldwide populations. Endocrinol Metab Clin North Am. 2004;33:351-376.

12 Schillaci G, Pirro M, Vaudo G, et al. Prognostic value of the metabolic syndrome in essential hypertension. J Am Coll Cardiol. 2004;43:1817-1822.

13 Isomaa B, Almgren P, Tuomi T, et al. Cardiovascular morbidity and mortality associated with the metabolic syndrome. Diabetes Care. 2001;24:683-689.

14 Cuspidi C, Meani S, Fusi V, et al. Metabolic syndrome and target organ damage in untreated essential hypertension. J Hypertens. 2004;22:1991-1998.

152003 European Society of Hypertension -European Society of Cardiology guidelines for the management of arterial hypertension. J Hypertens. 2003;21:1011-1053.

16 Grundy SM, Hansen B, Smith SC Jr, et al. Clinical management of metabolic syndrome: report of the American Heart Association/National Heart, Lung, and Blood Institute/American Diabetes Association conference on scientific issues related to management. Circulation. 2004;109:551-556.

17 Ford ES, Giles WH, Dietz WH. Prevalence of the metabolic syndrome among US adults: findings from the third National Health and Nutrition Examination Survey. JAMA. 2002;287:356-359.

18 Rutter MK, Meigs JB, Sullivan LM, et al. C-Reactive protein, the metabolic syndrome, and prediction of cardiovascular events in the Framingham offspring study. Circulation. 2004;110:380-385.

19 Sattar N, Gaw A, Scherbakova O, et al. Metabolic syndrome with and without C-reactive protein as a predictor of coronary heart disease and diabetes in the West of Scotland Coronary Prevention Study. Circulation. 2003;108:414-419.

20 Ridker PM, Buring JE, Cook NR, et al. C-reactive protein, the metabolic syndrome, and risk of incident cardiovascular events: an 8-year follow-up of 14,719 initially healthy American women. Circulation. 2003;107:391-397.

21 Martinez-Larrad MT, Fernández-Perez C, González-Sanchez JL, et al. Prevalence of the metabolic syndrome (ATP-III criteria). Population-based study of rural and urban areas in the Spanish province of Segovia. Med Clin (Barc). 2005;125:481-486.

22 Álvarez EE, Ribas L, Serra LL. Prevalence of the metabolic syndrome in the population of Canary Islands, Spain. Med Clin (Barc). 2003;120:172-174.

23 Ascaso JF, Romero P, Real JT, et al. Abdominal obesity, insulin resistance and metabolic syndrome in a southern European population. Eur J Intern Med. 2003;14:101-106.

24 Alegria E, Cordero A, Grima A, et al. Prevalence of metabolic syndrome in the Spanish working population MESYAS registry. Rev Esp Cardiol. 2005;58:797-806.

25 De La Sierra A, Romero R, Bonet J, et al. Prevalence and general features of the metabolic syndrome in the Spanish hypertensive population. Med Clin (Barc). 2006;126:406-409.

26 Gimeno JA, Lou JM, Molinero E, et al. Metabolic syndrome as a cardiovascular risk factor in patients with type 2 diabetes. Rev Esp Cardiol. 2004;57:507-513.

27 Hernandez A, Riera C, Solá E, et al. Prevalence of metabolic syndrome in patients with coronary heart disease. Med Clin (Barc). 2003;121:204-208.

28 Kanauchi M, Kanauchi K, Hashimoto T, et al. Metabolic syndrome and new category 'pre-hypertension' in a Japanese population. Curr Med Res Opin. 2004;20:1365-1370.

29 Egan BM, Papademetriou V, Wofford M, et al. Metabolic syndrome and insulin resistance in the TROPHY substudy: contrasting views in patients with high-normal blood pressure. Am J Hypertens. 2005;18:3-12. 
30 Ford ES. Prevalence of the metabolic syndrome defined by the International Diabetes Federation among adults in the U.S. Diabetes Care. 2005;28:2745-2749.

31 Barrios V, Campuzano R, Peña G, et al. Cardiovascular risk stratification in hypertensive patients attended in primary care setting and its impact on the antihypertensive treatment. The DIORISC study. Hipertensión. 2002;19:114-120.

32 Backlund L, Bring J, Strender L-E. How accurately do general practitioners and students estimate coronary risk in hypercholesterolaemic patients? Prim Health Care Res Dev. 2004;5:145-152.

33 Listerri JL, Rodriguez GC, Alonso FJ, et al. Blood pressure control in Spanish hypertensive patients in Primary Health Care Centres. PRESCAP 2002 Study. Med Clin (Barc). 2004;122:165-171. 